\title{
WILLINGNESS TO SHARE PERSONAL INFORMATION
}

\author{
Lilly Elisabeth Both \\ Department of Psychology, University of New Brunswick (Canada)
}

\begin{abstract}
The purpose of this study was to examine the factors that influence an individual's choice to share personal information online. Specifically, the role of age, gender, personality, overall media exposure, and perceived risks and benefits were examined in relation to a willingness to share personal information that differed in sensitivity (high school grades, medical records, income) and differed in target audience (social media, online store, general public). A total of 202 individuals participated in this survey study. The majority were young $(M$ age $=22.46$ years, $S D=5.77)$, single $(83.7 \%)$, women $(80.7 \%)$, with at least some post-secondary education $(90.1 \%)$. A series of hierarchical regression analyses were conducted. The results indicated that willingness to share personal information on social media was predicted by having higher scores on the personality traits of extraversion, agreeableness, and negative emotionality. Higher scores on perceived purchase benefits and total media exposure also predicted willingness to share personal information on social media. In terms of willingness to share personal information with an online store, total media exposure was a significant predictor along with higher extraversion and lower conscientiousness scores. Finally, willingness to share personal information with the general public was predicted by overall media exposure. Participants generally believed that there were risks involved in sharing personal information, but these risks were considered to be slight. As well, they only slightly disagreed when asked if the internet could be trusted, and were neutral on whether there were purchase benefits to providing personal information.
\end{abstract}

Keywords: Privacy, personal information, personality, social media use.

\section{Introduction}

E-commerce transactions have become increasingly more popular during the global COVID-19 pandemic. To provide services, online stores require personal information such as credit card numbers, addresses and names. However, increasingly, people are willing to share personal information on other platforms such as social media, where access to this private information is not always necessary.

\subsection{Purpose of the present study}

The purpose of this study was to examine factors that influence an individual's choice to share personal information online. Specifically, the role of age, gender, personality, overall media exposure, and perceived risks and benefits were examined in relation to a willingness to share personal information that differed in sensitivity (high school grades, medical records, income) and differed in target audience (social media, online store, general public).

\section{Method}

\subsection{Participants}

The participants consisted of 202 adults between the ages of 19.0 and 54.4 years. The majority were young $(M$ age $=22.46$ years, $S D=5.77)$. In this sample, $80.7 \%$ identified as being women, $17.8 \%$ as men, and $1.5 \%$ as transgender. In terms of marital status, $83.7 \%$ were single, $14.8 \%$ were married or living common law, and $1.5 \%$ were divorced. Participants were mainly Caucasian $(88.6 \%)$ and educated ( $90.1 \%$ had completed at least some university or community college courses). Participants were recruited through announcements in psychology courses at the university, and a link to the online study could be shared with others via social media. 


\subsection{Measures}

Demographic Questionnaire. Participants were asked a series of questions regarding age, gender, marital status, education level and ethnicity.

The Big Five Inventory - 2 (BFI-2; Soto \& John, 2017). The BFI-2 is commonly used to measure five personality traits: Negative Emotionality (or neuroticism), Extraversion, Open-mindedness, Agreeableness, and Conscientiousness. In this study, the internal reliability was excellent (Cronbach's a = .85 for Extraversion; .79 for Agreeableness; .87 for Conscientiousness; .91 for Negative Emotionality; and .82 for Open-Mindedness).

Purchase Benefits Survey (Robinson, 2018). Robinson (2018) adapted this scale based on purchase benefit questions from Gupta, Iyer and Weisskirch (2010). The measure consists of five questions rated on a 7-point scale where $1=$ not at all important to $7=$ extremely important. An overall score was computed, and the scale had excellent internal reliability in this study (Cronbach's a $=.85$ ).

Risk Beliefs Scale (Malhotra, Kim \& Agarwal, 2004). Malhotra et al. (2004) adapted this scale from Jarvenpaa, and Tractinsky (1999). The Risk Beliefs Scale consists of four questions rated on a 7-point scale from $1=$ strongly disagree to $7=$ strongly agree. A total score was computed for this scale, and the internal reliability of Cronbach's a $=.86$ was excellent.

Trust in the Internet (Robinson, 2018). Robinson (2018) adapted this scale based on internet trust questions from Dinev and Hart (2006). The scale consists of four questions rated on a 7-point scale where $1=$ strongly disagree to $7=$ strongly agree. An overall score was computed, and the scale had excellent internal reliability in this study (Cronbach's a $=.82$ ).

Media Exposure Scale. For the purpose of this study, three questions asked participants how often they shop online, bank online and use Apps that ask for personal information. These questions were rated on a scale from $1=$ never, 2 =rarely, $3=$ occasionally, $4=$ frequently, and $5=$ very frequently. The scores from the three questions were summed to give an overall total score of media exposure.

Willingness to Share Information (adapted from Shubert et al., 2018). Shubert et al. (2018) examined how willing people were to share personal data. They varied the sensitivity of information (such as high school grades, medical records, last year's tax return, gender, education, ethnicity, current location, address) in three domains (a social media website, an online store, a broader public). These questions and the format were adapted for the present study. The three domains were used, but the sensitivity of information questions were broadened to include more items such as bank account balance, credit card information, phone number, drivers' license photo, etc. Participants rated how likely they would be willing to share their personal information for each of these questions in each of the three domains. The items were rated on a scale of $1=$ extremely unlikely to $5=$ extremely likely. In total, 24 questions were asked in each domain, and an overall average score was computed for each domain. Cronbach's $\mathrm{a}=.88$ for the 24 items comprising the willingness to share information on a social media platform like Facebook or Twitter; Cronbach's a $=.88$ for sharing information with an online store; and Cronbach's $\mathrm{a}=.92$ for sharing information with the general public.

\subsection{Procedure}

Participants were recruited from psychology courses at the university. They read a description of the study and were directed to Qualtrics, an online survey platform. A consent form and the demographic measure were always presented first, followed by the remaining questionnaires in random order. The entire survey took about 40 minutes to complete and students could earn one bonus point toward their final grade (students had the option of earning bonus points through other means if they did not wish to participate in research). The survey link could also be shared on social media platforms. As an incentive to participate, all participants also had the option of being entered into a draw for a $\$ 50$ Amazon gift card.

\section{Results}

Participants only slightly agreed ( $M=5.1$ on a 7-point scale) that there were risks involved in sharing personal information. When asked if the internet could be trusted, they only slightly disagreed $(M$ $=3.3$ on a 7-point scale), and they were neutral on whether there were purchase benefits ( $M=4.4$ on a 7 point scale).

\subsection{Hierarchical regression analyses}

Three hierarchical regression analyses were conducted predicting: willingness to share personal information on social media; willingness to share personal information with an online store; and willingness to share personal information with the broader public. For each of these criterion variables, age and gender were entered on the first step. The five personality factors of Negative Emotionality, Extraversion, Open-Mindedness, Agreeableness, and Conscientiousness were added on the second step. 
Finally, purchase benefits, risk beliefs, trust in the internet, and media exposure were added on the third step. For each of these hierarchical regression analyses, Tolerance and VIF were within acceptable levels.

Willingness to share personal information on social media. The overall model was statistically significant and accounted for $25 \%$ of the variance on the measure of willingness to share personal information on social media $(F(11,185)=5.66, p<.001$, multiple $R=.50)$. Age and gender were not statistically significant predictors. Significant predictors were Extraversion $(\beta=.20)$, Agreeableness $(\beta=.21)$, Negative Emotionality $(\beta=.21)$, purchase benefits $(\beta=.17)$, and media exposure $(\beta=.31)$.

Willingness to share personal information with an online store. The overall model was statistically significant and accounted for $16 \%$ of the variance on the measure of willingness to share personal information with an online store $(F(11,186)=3.17, p=.001$, multiple $R=.40)$. Age and gender were not statistically significant predictors. Significant predictors were Extraversion $(\beta=.20)$, and Conscientiousness $(\beta=-.19)$ along with media exposure $(\beta=.22)$. The adjusted $R^{2}$ value of .11 in the overall model indicates that only $11 \%$ of the variability in the willingness to share personal information with an online store was predicted by higher scores on Extraversion and media exposure, and lower scores on Conscientiousness.

Willingness to share personal information with a broader public. The overall model was statistically significant and accounted for $12 \%$ of the variance in willingness to share personal information with a broader public $(F(11,186)=2.29, p=.012$, multiple $R=.35)$. The only significant predictor was media exposure $(\beta=.16)$.

\section{Discussion}

Age and gender did not predict willingness to share personal information; media exposure was the only consistent predictor across the different platforms. The more media exposure participants had, the more willing they were to share personal information. Although people understood there were risks involved, they felt these risks were slight. They only slightly disagreed when asked if the internet could be trusted. Participants were more willing to share information on social media than they were with an online store, and were least willing to share information with the broader public. Further research is needed to address why people would provide sensitive information to others in cases where it isn't necessary.

\section{References}

Dinev, T., \& Hart, P. (2006). An extended privacy calculus model for e-commerce transactions. Information Systems Research, 17(1), 61-80.

Gupta, B., Iyer, L.S., \& Weisskirch, R.S. (2010). Facilitating global e-commerce: A comparison of consumers' willingness to disclose personal information online in the US and in India. Journal of Electronic Commerce Research, 11(1), 41-52.

Jarvenpaa, S.L., \& Tractinsky, L.N. (1999). Consumer trust in an internet store: A cross-cultural validation. J. Comput.-Mediated Comm., 5(2). Retrieved November 3, 2018, from http://www.ascosc.org/jcmc/vol5/issue2/jarvenpaa.html

Malhotra, N.K., Kim, S.S., \& Agarwal, J. (2004). Internet Users' Information Privacy Concerns (IUIPC): The construct, the scale, and a causal model. Information Systems Research, 15, 336-355.

Robinson, S.C. (2018). Factors predicting attitude toward disclosing personal data online. Journal of Organizational Computing and Electronic Commerce, 28(3), 214-233. doi: 10.1080/10919392.2018.1482601

Shubert, R., Koumoutsakos, P., Arampatzis, G., Wang, Y., Hug, F., \& Marinica, I. (2018). Are people willing to share their personal data? Insights from two survey studies. Working Paper No. 1. Collegium Helveticum. Retrieved November 3, 2018, from https://collegium.ethz.ch /wpcontent/uploads/2018/07/180705 are people willing.pdf

Soto, C.J., \& John, O.P. (2017). The next Big Five Inventory (BFI-2): Developing and assessing a hierarchical model with 15 facets to enhance bandwidth, fidelity, and predictive power. Journal of Personality and Social Psychology, 113(1), 117-143. 